\title{
EVALUACIÓN FORMATIVA Y COMPARTIDA EN EL PRIMER CURSO DE DOBLE GRADO DE EDUCACIÓN INFANTIL Y EDUCACIÓN PRIMARIA. LOS TRABAJOS DE APRENDIZAJE TUTORADO COMO EJEMPLO DE BUENA PRÁCTICA
}

Formative and shared assessment in first year of the double degree of Pre-service Teacher Education. The Tutored Learning Projects as an example of good practice Avaliação formativa e partilhada no primeiro ano da Licenciatura dupla em educação pré-escolar e educação primária. Os trabalhos de aprendizagem tutorado como exemplo de boas práticas

\section{Miriam Molina Soria (1) \\ Cristina Pascual Arias (2)}

Víctor Manuel López Pastor (3)

(1) Universidad de Valladolid, España. Teléfono: +34 921112321. Correo electrónico: miriam.molina@uva.es

(2) Universidad de Valladolid, España. Teléfono: +34 921112321. Correo electrónico: cristina.pascual@uva.es

(3) Universidad de Valladolid, España. Teléfono: +34 921112321. Correo electrónico: vlopez@mpc.uva.es

\section{Resumen}

Este trabajo presenta una experiencia de buena práctica de Evaluación Formativa y Compartida (EFyC) en el primer curso del doble grado de educación infantil y educación primaria. Se trata de un Proyecto de Aprendizaje Tutorado (PAT) utilizado en la Formación Inicial del Profesorado (FIP), durante el segundo cuatrimestre del curso 2018/19, en la asignatura de Fundamentos y Didáctica de la Educación Corporal Infantil. Los resultados han sido positivos para el alumnado, gracias a la metodología de aprendizaje y al sistema de EFyC llevada a cabo. Se obtienen valores muy altos en rendimiento y éxito académico. La carga de trabajo del alumnado está dentro de las horas oficialmente previstas de trabajo no presencial, por lo que no puede ser considerada como excesiva.

Palabras clave: Proyectos de aprendizaje tutorado, formación inicial del profesorado; evaluación formativa; evaluación compartida; Educación Infantil 


\begin{abstract}
This work presents a good practice experience of Formative and Shared Assessment (F\&SA) in the first year of double degree of Pre-service Teacher Education. It is a Tutored Learning Project (TLP) used in Pre-service Teacher Education (ITE), during the second semester of the 2018/19 school year, in the subject of Physical Education in Childhood Education. The results have been positive for the students, thanks to the learning methodology and the F\&SA system carried out. Very high values are obtained in performance and academic success. The workload of the students is within the officially foreseen hours of non-contact work, so it cannot be considered as excessive.

Keywords: Tutored learning projects, pre-service teacher education; formative assessment; shared assessment; early childhood education
\end{abstract}

\title{
Resumo
}

Este trabalho apresenta uma experiência de boas práticas de avaliação formativa e partilhada (AFyP) no primeiro ano da Licenciatura dupla em educação pré-escolar e educação primária. Trata-se de um Projeto de Aprendizagem Tutorado (PAT), utilizado na Formação Inicial de Professores (FIP), durante o segundo quadrimestre do ano letivo 2018/19, na disciplina Fundamentos e Didática da Educação Corporal Pré-escolar. Os resultados foram positivos para os alunos, graças à metodologia de aprendizagem e ao sistema AFyP usado. Foram obtidos valores muito altos ao nível do desempenho e sucesso académico. A carga de trabalho dos alunos não pode ser considerada excessiva, porque está dentro das horas, oficialmente previstas, de trabalho não presencial.

Palavras-chave: Projetos de aprendizagem tutorado, formação inicial de professores; avaliação formativa; avaliação partilhada; educação pré-escolar

\section{Introducción}

La evaluación formativa y compartida (EFyC) se puede definir como un proceso de evaluación que tiene como finalidad mejorar los procesos de enseñanza-aprendizaje (López-Pastor, 2009; López-Pastor \& Pérez-Pueyo, 2017). Por esta razón, se aplican esos sistemas de evaluación a los trabajos de nuestra asignatura. 
El ejemplo de buena práctica que presentamos en esta comunicación es denominado "Proyectos de Aprendizaje Tutorados" (PAT), que han demostrado ser una forma muy eficaz de trabajo teórico-práctico y de desarrollo de competencias profesionales.

El PAT consiste en una actividad de aprendizaje grupal que, normalmente, implica la realidad de una práctica (real o simulada), donde se ponen en juego diferentes competencias profesionales (Barba, López, Manrique, Gea \& Monjas, 2010; Manrique et al., 2010).

\section{Contextualización}

En nuestro centro llevamos muchos años utilizando la metodología de Proyectos de Aprendizaje Tutorados (PAT), porque han demostrado ser una forma muy eficaz de trabajo teórico-práctico y ayudan a desarrollar las competencias profesionales. El PAT es una actividad grupal: cada grupo prepara una sesión práctica y un marco teórico de un tema a elegir dentro de una lista. Se ofrecen al alumnado propuestas de intervención para educación infantil al inicio de la asignatura.

La EFyC se da durante todo el proceso de elaboración y puesta en práctica del PAT. Comienza en las tutorías previas profesorado-alumnado para la revisión y mejora de documentos y plan de actuación, se da de forma importante durante la puesta en práctica, y se termina en los diferentes informes finales que elabora el alumnado, hasta cerrar el proceso de forma satisfactoria. La EFyC demuestra ser un proceso clave para lograr mejores aprendizajes y conseguir una buena calidad de los trabajos.

Esta experiencia se lleva a cabo en la Facultad de Educación de Segovia (Universidad de Valladolid), en $1^{\mathrm{o}}$ de doble grado de Educación Infantil y Primaria, asignatura de Fundamentos y Didáctica de la Educación Corporal Infantil, durante el segundo cuatrimestre del curso 2018/19. Esta asignatura tiene 6 créditos ECTS: 60 horas lectivas y 90 horas de trabajo no presencial. Es un grupo de 50 alumnas y alumnos.

\section{Diseño y desarrollo}

El proceso de construcción del PAT tiene las siguientes fases, explicadas en la Tabla 1:

\section{Evaluación}

En la tabla 2 se presentan las diferentes actividades de aprendizaje de la asignatura, con los procesos de evaluación de cada una y el porcentaje de la calificación final. 
Tabla 1.

Fases en la realización del PAT

\begin{tabular}{|c|c|}
\hline & Fases \\
\hline 1 & Cada grupo elige un tema y se asigna una fecha de realización para cada grupo. \\
\hline 2 & $\begin{array}{l}\text { En una primera tutoría se explica a cada grupo los documentos básicos que deben leer según el } \\
\text { tema para elaborar un marco teórico. }\end{array}$ \\
\hline 3 & $\begin{array}{l}\text { Cada grupo realiza un primer borrador del marco teórico del tema, de unas cuatro páginas, y un } \\
\text { plan de sesión. }\end{array}$ \\
\hline 4 & Acuden a tutorías para que el profesor corrija los borradores y explica aspectos a mejorar. \\
\hline 5 & Se realizan las tutorías necesarias hasta que los documentos tienen buena calidad. \\
\hline 6 & $\begin{array}{l}\text { Se realiza la sesión práctica y se expone el marco teórico (en diez minutos). El grupo encargado } \\
\text { de dicho tema da una copia a cada compañero. }\end{array}$ \\
\hline 7 & Es opcional que cada grupo lleve a cabo el PAT en un centro de Educación Infantil. \\
\hline 8 & $\begin{array}{l}\text { Una semana después cada grupo debe entregar el informe final del PAT. En este informe se } \\
\text { reflexiona sobre la puesta en práctica del trabajo y se realiza una autoevaluación. }\end{array}$ \\
\hline 9 & $\begin{array}{l}\text { Si el profesor considera que el informe es correcto, lo devuelve para que lo guarden en su carpeta. } \\
\text { Si hay aspectos a mejorar deben corregirlo y entregarlo en un plazo máximo de una semana. }\end{array}$ \\
\hline
\end{tabular}

Tabla 2.

Resumen de actividades de aprendizaje, evaluación y peso en la calificación final.

\begin{tabular}{|c|c|c|c|}
\hline Actividad de aprendizaje & Instrumento evaluación & Evaluación formativa & $\begin{array}{c}\text { Peso en } \\
\text { calificación }\end{array}$ \\
\hline 1-PAT & $\begin{array}{l}\text { Escala descriptiva } \\
\text { (rúbrica) }\end{array}$ & \multirow{5}{*}{$\begin{array}{l}\text { En todos los casos cada } \\
\text { documento entregado se } \\
\text { revisa por el profesorado, } \\
\text { aportando feed-back para } \\
\text { su mejora y realizando } \\
\text { procesos de evaluación } \\
\text { compartida a través de las } \\
\text { fichas de autoevaluación. } \\
\text { Si hay cosas que tengan } \\
\text { que ser corregidas, se da } \\
\text { una semana de plazo para } \\
\text { hacerlo. }\end{array}$} & 30 \\
\hline $\begin{array}{l}\text { 2-Fichas de sesiones } \\
\text { prácticas }\end{array}$ & $\begin{array}{l}\text { Escala descriptiva } \\
\text { (rúbrica) }\end{array}$ & & 20 \\
\hline $\begin{array}{l}\text { 3-Examen parcial con co- } \\
\text { evaluación inmediata }\end{array}$ & Planilla & & 20 \\
\hline $\begin{array}{l}\text { 4-Mapas conceptuales y } \\
\text { dossier asignatura }\end{array}$ & $\begin{array}{l}\text { Escala descriptiva } \\
\text { (rúbrica) }\end{array}$ & & 10 \\
\hline $\begin{array}{l}\text { 5-Lecturas y tertulias } \\
\text { dialógicas }\end{array}$ & $\begin{array}{l}\text { Escala descriptiva } \\
\text { (rúbrica) }\end{array}$ & & 20 \\
\hline
\end{tabular}

La evaluación de la asignatura se lleva a cabo a través de un cuestionario anónimo con escala tipo Likert (1-5) y dos escalas de autopercepción de competencia del alumnado con escala tipo Likert (1-4). Actualmente sólo disponemos de los datos de la escala pretest de autopercepción de competencia. Los presentamos en los anexos I, II y III.

Los datos del Anexo I muestran las medias en autopercepción de competencias transversales, con valores generalmente altos, en torno al 3.00 en la mayoría de ellos, oscilando entre 2.78 (conocer una lengua extranjera) y 3.34 (desarrollar un compromiso ético).

Los datos del Anexo II muestran las medias en competencias docentes generales, con valores altos, pero ya más bajos que la tabla anterior. La mayoría de los ítems se sitúan 
en valores cercanos a 2.6, oscilando entre 2.28 (poner en marcha procesos de investigación en el aula) y 2.94 (implicar al alumnado en su aprendizaje).

Los datos del Anexo III muestran las medias en competencias docentes específicas de $\mathrm{EF}$, con valores entre medios y altos, pero los más bajos de las tres tablas. La mayoría de los ítems se sitúan en valores cercanos a 2.4, oscilando entre 2.00 (conocer y aplicar los fundamentos biológicos, fisiológicos, de maduración evolutiva y el desarrollo psicomotor) y 3.03 (saber utilizar el juego como recurso didáctico y como contenido de enseñanza).

La escala postest se aplica a finales de mayo, por lo que no podremos compararlas hasta entonces.

\section{Conclusiones}

El proceso de EFyC se lleva a cabo durante toda la asignatura: el alumnado realiza las actividades correspondientes y las autoevalúa; a continuación, son evaluadas por los profesores y devueltas en el plazo de una semana con feedback que ayude a la mejora de los trabajos. Esta forma de trabajar ayuda al proceso de enseñanza-aprendizaje y ayuda a la adquisición de conocimientos.

Los datos del cuestionario de autopercepción de competencias nos indican que los alumnos se sienten bastante competentes en las tres escalas, aunque más en competencias transversales, seguida de las competencias docentes generales y las competencias específicas de EF. La pequeña diferencia existente entre los dos tipos de competencias docentes puede deberse a que son alumnos de primer curso, en su segundo cuatrimestre de carrera, por lo que todavía no han trabajado ninguna de las dos competencias.

En estos momentos no disponemos de los datos de carga de trabajo, ni de rendimiento, ni del cuestionario de evaluación de la asignatura, dado que esos datos se recogen al finalizar, en junio. Hasta el momento la asignatura está teniendo un proceso correcto, similar a cursos anteriores, por lo que parece razonable que los resultados sean similares.

\section{Referencias}

Barba-Martín, J. J., López-Pastor, VM., Manrique-Arribas, JC., Gea-Fernández, JM., \& Monjas-Aguado, R. (2010). Garantir l'èxit en la formació inicial del professorat 
d'educació física: els projectes d'aprenentatge tutelats. Temps d'educació, 39, 187-206.

López-Pastor, V. M. (coord.) (2009). Evaluación Formativa y Compartida en Educación Superior. Narcea: Madrid.

López-Pastor, V. M., \& Pérez-Pueyo, A. (coords.) (2017). Evaluación formativa y compartida en educación: experiencias de éxito en todas las etapas educativas. León: Universidad de León. (e-book). Recuperado de https://buleria.unileon.es/handle/10612/5999

Manrique Arribas, J.C., López Pastor, V.M., Monjas Aguado, R., \& Real Rubio, F. (2010). El potencial de los proyectos de aprendizaje tutorado y los sistemas de evaluación formativa en la mejora de la autonomía del alumnado. Una experiencia interdisciplinar en formación inicial del profesorado. Revista Española de Educación Física y Deportes, 14, 39-57. 
Anexo I. Tabla 3.

Datos del cuestionario de autopercepción de competencias.

\begin{tabular}{lc}
\hline \multicolumn{1}{c}{ Bloque 1: competencias transversales } & Media \\
& aritmética \\
& 2.91 \\
11.- Analizar y sintetizar & 3.09 \\
12.- Organizar y planificar & 3.16 \\
13.- Comunicarse de forma oral y escrita & 3.00 \\
14.- Comunicarse gestual y corporalmente & 2.78 \\
15.- Conocer una lengua extranjera & 3.00 \\
16.- Utilizar las Tecnologías de la Información y la Comunicación en el ámbito de estudio & 3.19 \\
17.- Trabajar en equipo & 3.50 \\
18.- Desarrollar habilidades en las relaciones interpersonales (empatía, asertividad, respeto & 2.91 \\
y escucha) & 3.28 \\
19.- Desarrollar habilidades intrapersonales (autoestima, motivación y confianza en un & 3.34 \\
mismo) & 3.09 \\
110.- Razonar de forma crítica y reflexiva & 2.91 \\
111.- Desarrollar un compromiso ético & 2.94 \\
112.- Desarrollar un aprendizaje autónomo & \\
113.- Adaptarse a situaciones nuevas & \\
114.- Desarrollar la creatividad &
\end{tabular}

Anexo II. Tabla 4.

Datos del cuestionario de autopercepción de competencias.

\begin{tabular}{lc}
\hline \multicolumn{1}{c}{ Bloque 2: Competencias docentes } & Media \\
& aritmética \\
& 2.41 \\
21.- Conocer las características organizativas de os centros educativos & 2.47 \\
22.- Elaborar propuestas de cambio de la realidad educativa & 2.53 \\
23.- Diseñar situaciones de aprendizaje & 2.91 \\
24.- Animar situaciones de aprendizaje & 2.69 \\
25.- Gestionar la progresión de los aprendizajes & 2.75 \\
26.- Diseñar estrategias de atención a la diversidad & 2.72 \\
27.- Poner en práctica estrategias de atención a la diversidad & 2.94 \\
28.- Implicar al alumnado en su aprendizaje & 2.84 \\
29.- Implicar al alumnado en la vida del centro & 2.37 \\
210.- Participar en la gestión del Centro & 2.84 \\
211.- Informar a las familias sobre la evolución de su hijo/a & 2.84 \\
212.- Implicar a las familias en el aprendizaje de su hijo/a & 2.56 \\
213.- Implicar a las familias en la vida del centro & 2.87 \\
214.- Afrontar los deberes y dilemas éticos de la profesión & 2.81 \\
215.- Atender al propio desarrollo profesional docente (autoevaluación, lecturas, cursos, \\
jornadas, congresos, redes de trabajo) & 2.47 \\
216.- Desarrollar procesos de innovación educativa en el aula & 2.28 \\
217.- Poner en marcha procesos de investigación en el aula & \\
\hline
\end{tabular}

Evaluación formativa y compartida en el primer curso de doble grado de Educación Infantil y Educación 
Anexo III. Tabla 5.

Datos del cuestionario de autopercepción de competencias.

Bloque 3: competencias específicas docentes de educación física

31.- Diseñar, aplicar y analizar intervenciones didácticas en el área de Educación Física

Media

aritmética

32.- Diseñar, desarrollar y evaluar los procesos de enseñanza-aprendizaje relativos a la

actividad física y el deporte con atención a las Necesidades Específicas de Apoyo

Educativo

33.- Conocer y promover las diferentes manifestaciones motrices que forman parte de la cultura tradicional

34.- Conocer y aplicar los fundamentos biológicos, fisiológicos, de maduración evolutiva y

el desarrollo psicomotor

35.- Diseñar, modificar y/o adaptar al contexto educativo situaciones motrices orientadas al desarrollo y perfeccionamiento de las habilidades motrices

36.- Conocer elementos y fundamentos de la expresión corporal y la comunicación no verbal y su valor formativo y cultural

37.- Saber utilizar el juego como recurso didáctico y como contenido de enseñanza

38.- Conocer los fundamentos básicos de la iniciación deportiva escolar y diseñar tareas específicas para utilizarlos en el ámbito de la enseñanza

39.- Identificar los riesgos que se derivan para la salud de la práctica de actividades físicas inadecuadas

310.- Diseñar en un plan de hábitos de vida saludable (higiene y alimentación) y de práctica de- actividad física regular

311.- Saber aplicar los fundamentos (técnicas) de las actividades físicas en el medio natural

312.- Saber utilizar diferentes instrumentos de evaluación en el área de Educación Física

3.03

2.06

2.50

3.13.- Tener capacidad de reflexión sobre el proceso de enseñanza/aprendizaje y las distintas metodologías dentro de las clases de Educación Física

2.16

314.- Analizar y comunicar, de manera crítica y fundamentada, el valor de la actividad física y el deporte y sus posibilidades de contribuir al desarrollo y bienestar de las personas

Evaluación formativa y compartida en el primer curso de doble grado de Educación Infantil y Educación 